\title{
Innovación e investigación educativa en Información y Documentación en la Universidad española entre 1998 y 2008
}

\author{
AUTORAS: Raquel Gómez Díaz. Universidad de Salamanca. (rgomez@usal.es) ; María \\ del Carmen Agustín Lacruz. Universidad de Zaragoza. (cagustin@unizar.es ) ; Mónica \\ Izquierdo Alonso Universidad de Alcalá de Henares. (monica.izquierdo@uah.es)
}

\begin{abstract}
RESUMEN: El propósito de este trabajo es estudiar el recorrido de la investigación e Innovación educativa en el área de Información y Documentación en la universidad española entre 1998, fecha en la que arranca el proceso de Bolonia y 2008, año en que se implantan y comienzan a impartirse los primeros Grados en Información y Documentación. Para conseguir este objetivo nos proponemos analizar los planes y los programas de investigación que promueven la investigación e innovación educativa en Biblioteconomía y Documentación, los distintos agentes que la desarrollan así cómo los principales cauces de difusión de las investigaciones realizadas. El estudio se realiza rastreando las convocatorias de proyectos de innovación educativa en el ámbito estatal, autonómico y propio de cada universidad; identificando las instituciones y los organismos que efectúan esta investigación y analizando la literatura científica y los foros académicos y profesionales de divulgación y diseminación de los logros alcanzados. Entre los principales resultados destaca la elaboración de un estado de la cuestión comprensivo y actualizado de la investigación e innovación educativa en Información y Documentación en España orientado a la delimitación y caracterización de las líneas de mejora.
\end{abstract}

PALABRAS CLAVE: Innovación educativa. Investigación educativa. Información y Documentación. Universidad. España.

ABSTRACT: The aim of this paper is to study the route of the educational research and innovation in Information Sciences in Spanish universities from 1998, when it starts the process of Bologna and 2008, and begin to be implemented Grades given to the first Information and Documentation. To achieve this we propose to analyze the plans and research programs that promote educational innovation and research in Library and Information Science, the various actors in development and the main channels of dissemination of research. The study is tracking the calls for projects for educational innovation at the state level, and autonomy of each university, identifying the institutions and bodies which carry out such research and analyze scientific literature and academic forums and professional outreach and dissemination of achievements. The main results include the development of a comprehensive state of the art and updated research and educational innovation in Information and Documentation in Spain aimed at the delineation and characterization of lines of improvement.

KEY WORDS: Innovation. Educational research. Information Sciences. University Spain. 


\section{NTRODUCCIÓN}

Biblioteconomía y Documentación, responsable de la titulación de Grado en Información y Documentación, es la denominación de la disciplina y una de las áreas de conocimiento más jóvenes ${ }^{1}$ dentro de la universidad española. Este hecho determina no sólo los métodos y técnicas de investigación que utiliza, sino también los temas que centran su interés docente y científico. A diferencia de otros estudios de más antigua tradición académica, una de sus características más relevantes en el momento actual es que simultanea el afán por robustecer su estatus científico y la preocupación por mejorar la formación de los futuros profesionales.

Coincidiendo con el desarrollo del proceso de convergencia europea, que pone en valor el concepto de "competencia" y particularmente de "competencia docente" del profesorado, y los conceptos de "aprendizaje a lo largo de la vida" y "competencia informacional", se observa un interés emergente por analizar e investigar los procesos de enseñanza-aprendizaje como procesos de transferencia de conocimiento e información. Por tanto, se constata que la innovación educativa es uno de los centros de interés de la investigación en Información y Documentación. En él coinciden aspectos que involucran el método científico, la preocupación por la formación de profesionales y la aspiración a la innovación entendida como proceso de mejora que aporta calidad a todo conjunto.

\section{MARCO CONCEPTUAL: ¿QUÉ ENTENDEMOS POR INNOVACIÓN EDUCATI VA?}

En su sentido más práctico, innovación es toda acción de dotar a cualquier recurso con una nueva capacidad para producir "riqueza", o utilidad (Gallego Reinoso, 2005, p. 25). De forma más específica se puede considerar que innovación es todo proceso de creación o transformación orientado hacia la mejora de un producto, servicio, idea 0 práctica. Supone una renovación de cualquier hecho u objeto para que resulte más competitivo, útil, eficaz, eficiente y atractivo. Por tanto, es un valor añadido que proporciona un plus de calidad a las realidades o hechos que transforma.

La innovación se puede tipificar en función de criterios distintos. Dentro del ámbito educativo son relevantes, al menos aquellos que consideran el grado de novedad que aporta y los que hacen referencia a la propia naturaleza de la mejora efectuada.

En función de su grado de novedad, la innovación puede ser: a) gradual o progresiva, cuando se produce a través de pequeños cambios que de una manera aislada son poco significativos, pero acumulados pueden suponer mejoras notables, y b) radical 0 substancial, cuando supone la creación de nuevos productos o procesos e implica una ruptura con lo ya establecido.

\footnotetext{
${ }^{1}$ Información y Documentación es la denominación que adopta en España una titulación universitaria y que se fragua en torno a la elaboración y publicación de su "Libro Blanco". Esta denominación revela el horizonte hacia el que desea dirigirse un área de conocimiento (Biblioteconomía y Documentación), en cuyo origen pesa el enfoque disciplinar orientado hacia objetos de estudio definidos como soportes. La nueva denominación pone de manifiesto una visión que desplaza su eje de atención hacia los procesos y las actividades documentales. También evidencia el movimiento que se está produciendo desde una disciplina ubicada dentro de las Humanidades hacia otra más vinculada con las Ciencias Sociales

${ }^{2}$ Un análisis detallado de estos conceptos puede consultarse en Zabalza Beraza, Miguel Ángel (2003).
} 
En función de la naturaleza de la mejora efectuada, la innovación puede ser: a) tecnológica, si las herramientas y conocimientos asociados a ellas se utilizan para conseguir los cambios buscados $\mathrm{y}, \mathrm{b}$ ) organizativa, cuando se orienta hacia el perfeccionamiento de los procesos y las formas de trabajo de la institución.

La eficacia de los procesos de innovación depende, en gran medida, de que se planifiquen adecuadamente los objetivos, se interrelacionen las acciones y se comuniquen y difundan las mejoras logradas, considerando de forma global el contexto en el que se van a desenvolver todos los elementos involucrados.

El ciclo de la innovación se recoge en la Figura 1 y muestra la interconexión existente entre la idea de mejora, la acción de llevarla a cabo y la difusión del progreso alcanzado.

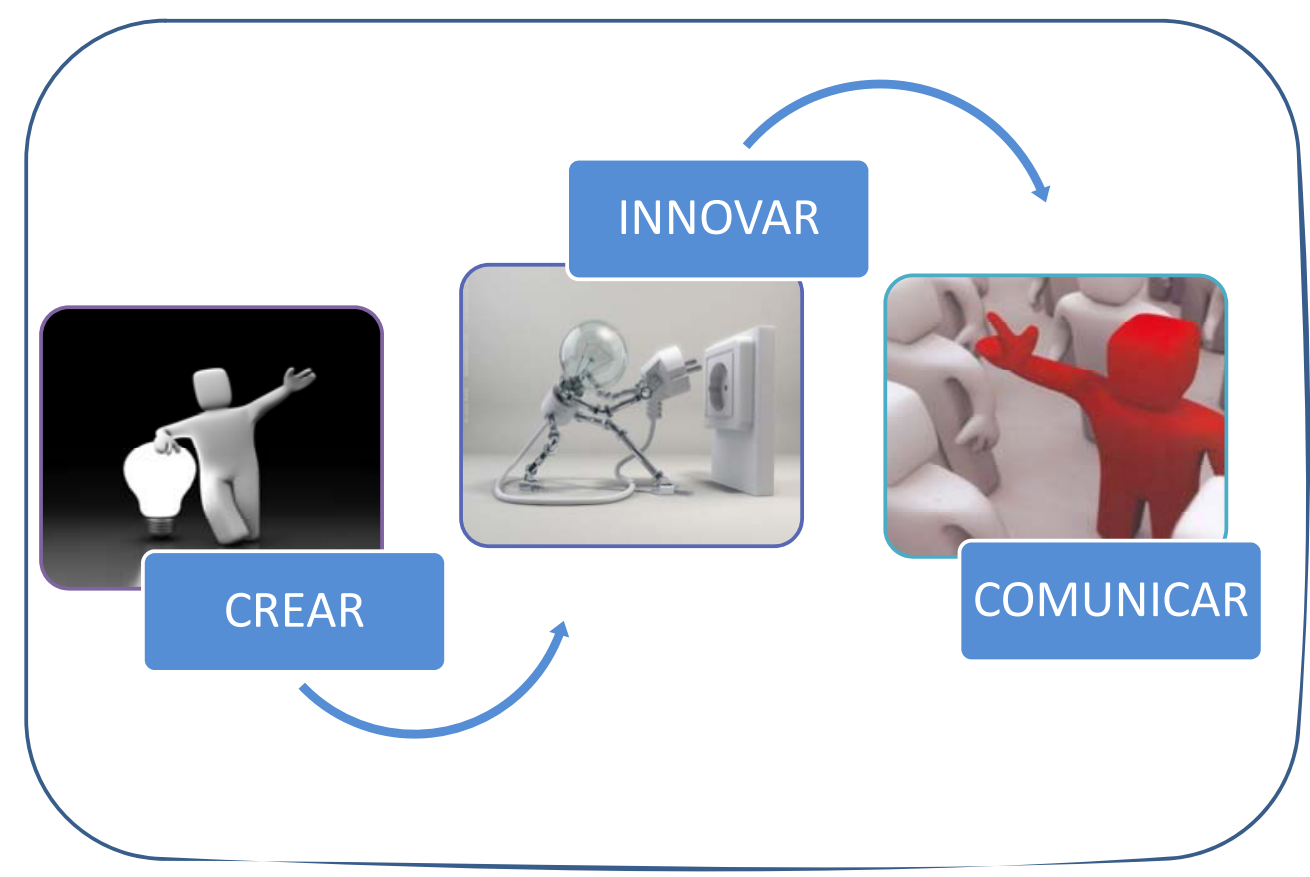

Fig. 1 Ciclo de la innovación

El concepto de innovación es uno de los más frecuentes y característicos de la reflexión actual acerca de la educación, aunque todavía no cuenta con un marco teórico específico y suficientemente consolidado. Aplicado a los procesos de enseñanzaaprendizaje se conoce como innovación educativa.

Esta denominación es sucesora de la expresión innovación docente, muy habitual en la bibliografía desde la década de los años ochenta y refleja que el centro de interés estaba focalizado en aquel momento hacia unas prácticas cuyo artífice era el profesorado.

El proceso de Bolonia, entre otros factores, ha favorecido una concepción más integradora del hecho educativo y un desplazamiento del eje de atención hacia otros agentes, como los estudiantes. Esto propicia que se extienda y amplíe la utilización de 
de la expresión innovación educativa, que se concibe no como una acción esporádica o improvisada, sino como el eje vertebrador del proceso de formación superior y se enmarca dentro de la planificación estratégica de cada una de las titulaciones desde la perspectiva de la búsqueda de la calidad.

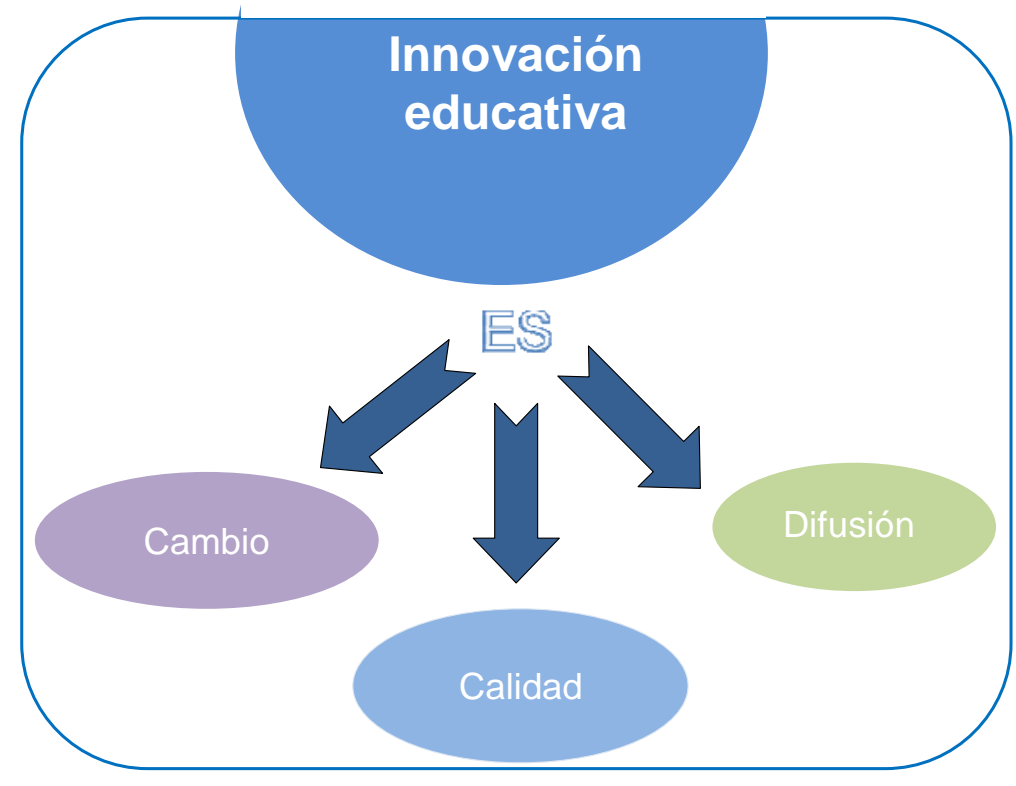

Fig. 2. Dimensión de la innovación educativa

A su vez, la calidad es también un concepto complejo y multidimensional cuyo significado se ha ido desdibujando a medida que se ha ido generalizando el uso de la palabra. Entendida en su dimensión educativa constituye una aspiración a la mejora efectiva del aprendizaje y la docencia, en cuya consecución intervienen numerosos elementos como "los valores [de la organización], el nivel de satisfacción, la acción formativa de los profesores, la cultura institucional, el clima de las clases, etc...." (Zabalza Beraza, 2003 p. 171).

El discurso de la calidad aplicado a la educación se desarrolla en parámetros no siempre coincidentes. De un lado se encuentra la calidad entendida como una la dimensión objetiva, real, constatable y sujeta a la medida, y de otro se encuentra su dimensión simbólica, la calidad concebida como mito, técnica de mercado o seña de identidad. En este sentido se ha convertido en un lema, en una realidad imaginada que pretende persuadir a los usuarios de que existe y por tanto conducirles hacia una acción determinada.

\section{INVESTIGACIÓN E INNOVACIÓN EDUCATIVA EN INFORMACIÓN Y DOCUMENTACI ÓN}

En Información y Documentación, la investigación en innovación educativa ha seguido, en buena medida, un recorrido paralelo al proceso de convergencia europea. Impulsado por él y bajo el paraguas de las nuevas tecnologías, muchos profesores, estudiantes, departamentos y universidades están modificando sus formas de aprender 
y enseñar y paralelamente, reflexionando con métodos científicos sobre cómo y para qué lo hacen. Es decir, están llevando a cabo procesos de innovación y mejora que contribuyen al desarrollo científico de la disciplina, como recoge la Figura 3.

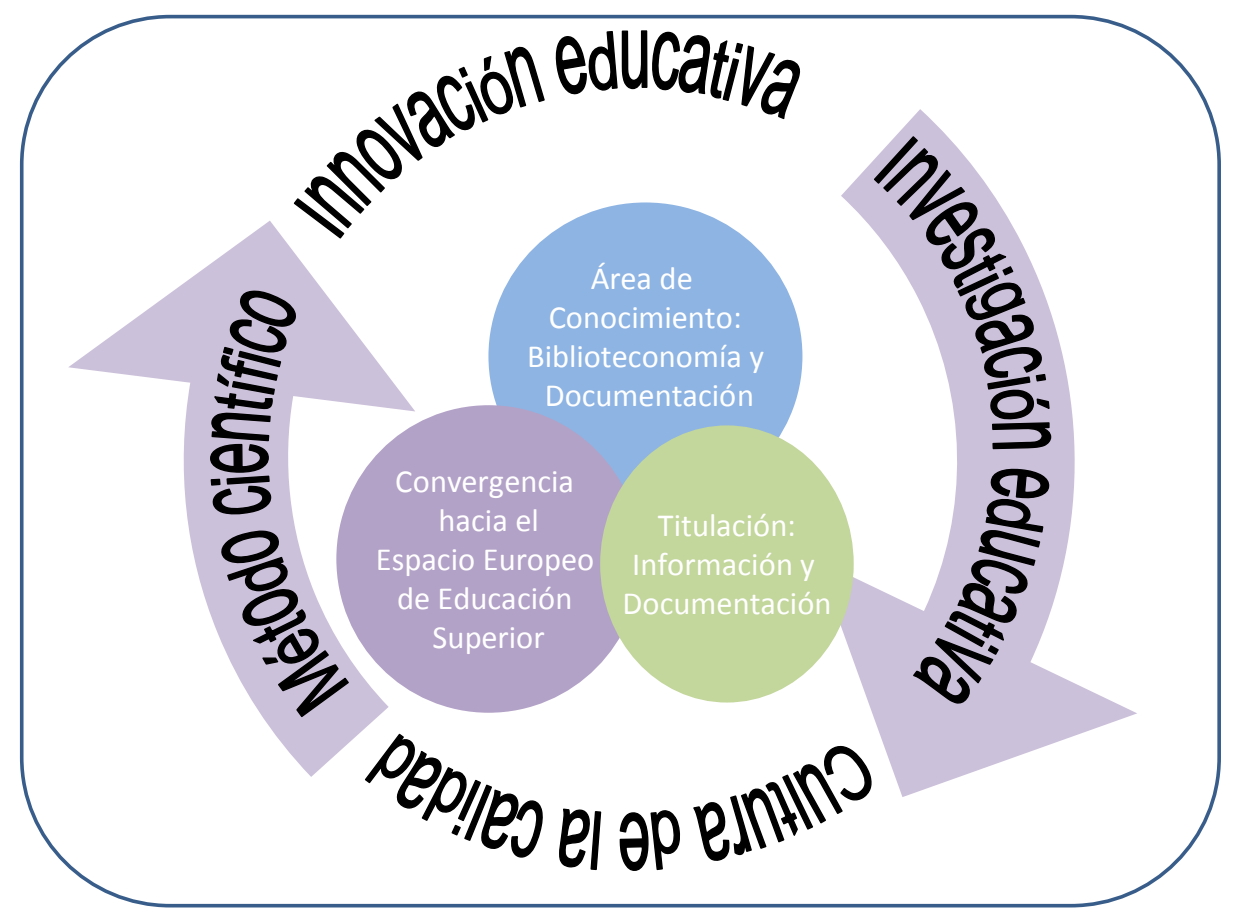

Fig. 3 Contexto de la innovación educativa en Información y Documentación

La innovación está desarrollándose en la actualidad dentro de las Ciencias de la Documentación siguiendo dos trayectorias. Por un lado se entiende que la innovación es un valor añadido que se incorpora a la actividad docente e investigadora del profesorado - la innovación como oficio- y por otro, la innovación entendida como horizonte característico de los saberes y las prácticas propias de la enseñanza universitaria - la innovación como cultura-. Ambas dimensiones conectan bien con el ideario de búsqueda de calidad y de adaptación continua a los nuevos entornos que busca la filosofía educativa actual.

La trayectoria recorrida permite tener una cierta panorámica de los logros alcanzados, no obstante, es necesario disponer de nuevas herramientas que permitan llevar a cabo la toma de las decisiones o plantear nuevas metas y etapas.

\subsection{Objetivo}

Con el propósito de elaborar un diagnóstico que permita establecer líneas de actuación e indicar las áreas de mejora este estudio pretende elaborar un estado de la cuestión comprensivo y global de la investigación en innovación educativa en el área de Biblioteconomía y Documentación, que incluya la identificación de las acciones de 
mejora, los fines que persiguen, los sujetos que las llevan a cabo y el estudio de los métodos, herramientas y procedimientos utilizados.

\subsection{Marco temporal}

El periodo estudiado abarca desde 1998 hasta 2008. La década elegida coincide con el inicio del proceso de convergencia hacia el Espacio Europeo de Educación Superior y se cierra con la implantación de los primeros grados en Información y Documentación en la Universidad española ${ }^{3}$.

Durante este periodo se han producido algunos hitos importantes como la publicación del Libro Blanco del título de Grado en Información y Documentación que ha favorecido la reflexión sobre la disciplina, al tiempo que ha permitido que los planes de estudio de la nueva titulación compartan unas metodologías educativas comunes. El marco teórico de la convergencia europea ha provocado una reflexión acerca de cómo articular la nueva enseñanza universitaria donde la innovación educativa está jugando un papel muy importante ${ }^{4}$.

\subsection{Método de estudio}

Para conseguir el objetivo trazado ha sido necesario identificar los Planes estratégicos de actuación, las Convocatorias y Programas, así como los proyectos concretos que promueven la investigación e innovación educativa en Biblioteconomía y Documentación; los distintos agentes que la desarrollan así cómo los principales cauces de difusión de las investigaciones realizadas.

Al ser la innovación educativa una línea estratégica, no vehiculada a través de convocatorias de rango estatal, sino autonómico, los objetivos y los formatos que adoptan las convocatorias son notablemente diversos. En este sentido hay que destacar que la naturaleza de las administraciones públicas españolas provoca que se promuevan políticas y acciones desde distintos ámbitos institucionales y en ocasiones incluso parece que los agentes involucrados se solapan. Además las Comunidades Autónomas pueden promover directamente las convocatorias a través de organismos distintos ${ }^{5}$, o bien trasferir los recursos a las propias universidades con carácter finalista para innovación, e incluso transferirlos otorgándoles a las instituciones académicas autonomía para que establezcan las líneas de actuación que consideren oportunas.

Este cúmulo de circunstancias dificulta notablemente la labor de rastreo, identificación y seguimiento de la innovación educativa.

\footnotetext{
${ }^{3}$ El grado en Información y Documentación ha sido uno de los primeros títulos adaptados en España al proceso de convergencia europea. Las Universidades de Zaragoza, Salamanca y Carlos III lo imparten desde el curso 2008-2009.

${ }^{4}$ Sobre esta cuestión véase la propuesta realizada por la Comisión formada para el estudio de la renovación de las metodologías educativas en las universidades españolas, cuyas conclusiones y recomendaciones se materializaron en la publicación del resumen ejecutivo titulado Propuestas para la renovación de las metodologías educativas en la Universidad.

${ }^{5}$ Los vaivenes que ha experimentado la innovación educativa como centro de interés científico también han estado relacionados con las diferentes sensibilidades asociadas a las orientaciones políticas.
} 
Por otra parte, a pesar de la importancia que adquiere la difusión en el ciclo de la investigación y la innovación, y de que muchas de iniciativas se comunican de manera adecuada, hay otras que tan sólo son conocidas por quienes las promueven y experimentan ${ }^{6}$. Este hecho provoca que aunque se procure la exhaustividad en la búsqueda de los resultados de estas acciones de mejora, muchas permanecen ocultas o poco visibilizadas por su falta de comunicación a través de los cauces habituales de la difusión científica?

\section{PRINCIPALES RESULTADOS DE LA INNOVACIÓN EDUCATIVA EN I NFORMACIÓN Y DOCUMENTACI ÓN}

A pesar de las dificultades señaladas en los párrafos anteriores es posible proponer una primera sistematización de la innovación educativa en nuestra área, que sin pretensiones de exhaustividad, resulte suficientemente representativa como para permitir conocer todo el circuito de la innovación educativa y plantear líneas e investigaciones futuras.

\section{a. Propósitos de la innovación}

El estudio de las evidencias documentales proporcionadas por las convocatorias y resoluciones publicadas, así como el análisis de las diferentes publicaciones en las que se difunde esta producción científica y académica, ponen de manifiesto que la investigación e innovación educativa se orienta principalmente en tres direcciones:

a) Diseño y aplicación de nuevas metodologías docentes:

- Diseño de módulos, materias asignaturas basados en competencias

- Implantación de metodologías activas de aprendizaje

- Desarrollo de sistemas de evaluación continua de competencias

- Desarrollo de nuevas modalidades de tutorización

- Diseño de programas de prácticas

b) Producción de materiales docentes y recursos didácticos de apoyo:

- Diseño de materiales multimedia

- Manuales

- Tutoriales

- Guías docentes

c) Realización de acciones de mejora, proyectos piloto y experiencias innovadoras

\section{b. Cauces de difusión}

El análisis de la información relativa a la producción académica y científica universitaria relacionada con el objeto estudiado, identifica dos canales de difusión principales:

\footnotetext{
${ }^{6}$ La difusión de la innovación es un elemento capital para la generación de nuevo conocimiento, como pone de manifiesto el Informe El papel de la información en la innovación (2006), donde una encuesta realizada sobre esta cuestión, constató que el $46 \%$ de los investigadores reconocen que la mayor parte de sus trabajos tienen su origen en otros trabajos previos, que les proporcionan nuevos enfoques.

${ }^{7}$ En el ámbito de la Información y la Documentación no se dispone por el momento de publicaciones periódicas especializadas en innovación educativa, ni tampoco hay otros congresos, seminarios o jornadas, distintos a los promovidos por EDIBCIC, que permitan conocer qué se ha hecho o se está haciendo sobre esta cuestión.
} 
a) Cauces formales:

- Publicaciones:

- Artículos científicos

- Monografías

- Contribuciones recogidas en actas de congresos, jornadas, encuentros, seminarios...

o Informes y otra literatura gris

- Celebración de seminarios, encuentros...

- Concesión de premios y distinciones

b) Cauces informales

- Páginas personales de profesores

- Listas de distribución

- Correos electrónicos

- Encuentros con colegas

\section{c. Agentes y destinatarios}

Es importante identificar los agentes y destinatarios de los procesos de innovación ya que son los sujetos que intervienen en ella quienes animan el proceso, al tiempo que constituyen la razón de ser que le da sentido.

La innovación educativa no es posible descontextualizada de aquellos por y para quienes ésta se planifica y lleva a cabo, aunque en ocasiones, la labor de identificación es compleja.

A continuación se propone una relación sistematizada de los agentes que intervienen (Figura 4):

a) Impulsores de las políticas educativas. Son los responsables del diseño e impulso de las líneas estratégicas de actuación. Se pueden categorizar en función de sus respectivos ámbitos de actuación.

- Estatales

o Ministros

o Secretarios de Estado

o Directores Generales, ...

- Autonómicos

o Consejeros

o Directores Generales, ...

- Universitarios ${ }^{8}$

o Rectores

o Vicerrectores

o Decanos y directores de centros

\footnotetext{
${ }^{8}$ las diferentes estructuras orgánicas de las universidades dificultan el seguimiento de los sujetos de la innovación, puesto que ésta pueda estar asignada como función o competencia a vicerrectorados, adjuntías, áreas, unidades, institutos u oficinas distintas. Además la forma de designarlas puede referirse específicamente tanto a la innovación educativa como de forma genérica al Espacio Europeo de Educación Superior, la Docencia, la Política educativa, el Profesorado e incluso la Calidad.
} 
b) Gestores académicos. Son los mediadores entre los impulsores de las políticas y los grupos de investigación. Se clasifican en:

- Directores de áreas, unidades e Institutos

- Vicedecanos, subdirectores,

- Coordinadores de titulaciones,

- Directores de departamentos, ...

c) Profesores, profesoras y personal de administración y servicios. Se organizan en equipos ad hoc, según los requisitos establecidos por las convocatorias, o bien forman parte de grupos de investigación estables, de carácter específicamente disciplinar o interdisciplinares.

d) Estudiantes. Constituyen uno de los ejes imprescindibles de los procesos de innovación educativa, aunque no suele ser frecuente contar con su participación explícita.

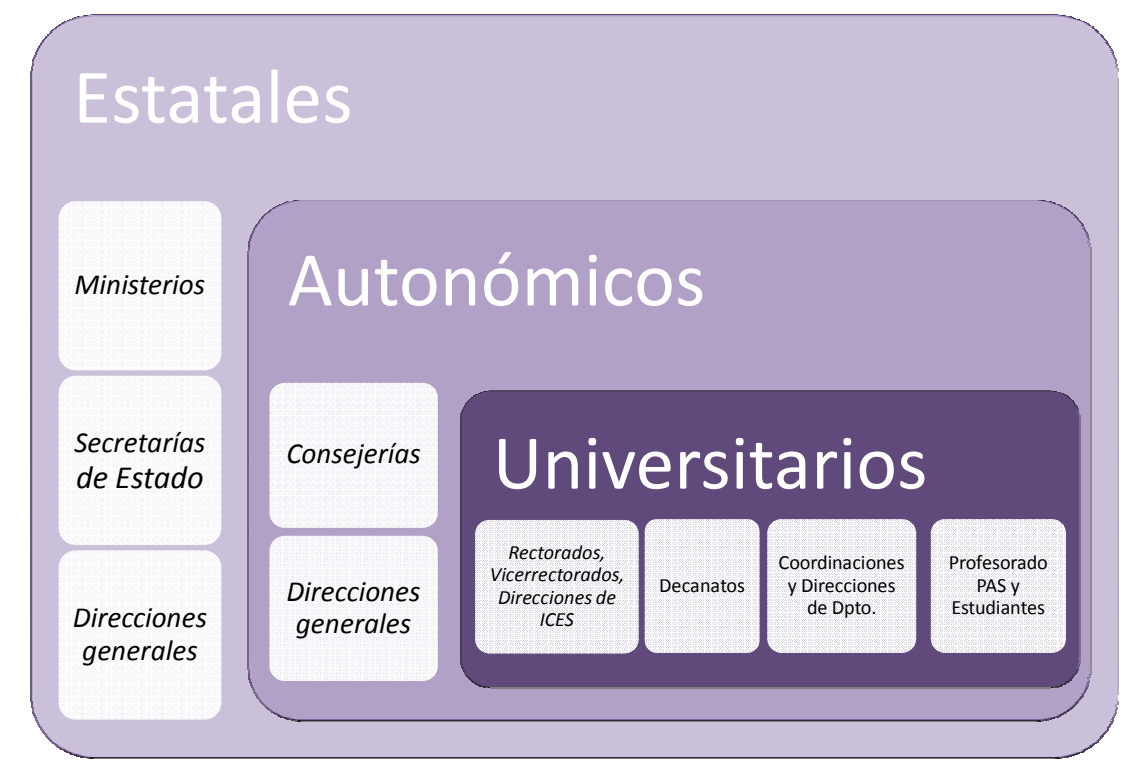

Fig. 4. Agentes de la innovación educativa

\section{d. Ámbito territorial}

Para completar el análisis de los elementos que intervienen en los procesos de innovación educativa es necesario determinar los diferentes espacios en los que ésta tiene lugar, pues las características contextuales definidas por los factores institucionales, académicos y geográficos condicionan los objetivos y los resultados de la innovación educativa.

Los ámbitos en los que ésta se desenvuelve se tipifican atendiendo a tres criterios:

a) Innovación desarrollada según el ámbito institucional:

- En Facultades, Centros e Institutos Universitarios

- En Departamentos

b) Innovación desarrollada según el ámbito académico 
- En titulaciones

- En asignaturas, materias y módulos

- En áreas de conocimiento

c) Innovación desarrollada según el ámbito geográfico (Figura 5)



Fig. 5 Mapa de las universidades españolas en las que se realiza innovación educativa en I y D

\section{CONCLUSI ONES}

El análisis llevado a cabo sobre la investigación e innovación educativa en el ámbito de la Información y Documentación en la universidad española en la década de 1998 a 2008 permite extraer las siguientes conclusiones:

1. La investigación en innovación educativa en Información y Documentación emplea los métodos, las técnicas y las herramientas propias de la actividad científica.

2. Sus resultados hacen posible la mejora de realidades, hechos y procedimientos anteriores, propiciando tanto nuevas formas de innovación, como el avance científico del Área de conocimiento.

3. La emergencia y el afianzamiento de la innovación educativa como objeto de interés específico de la actividad investigadora en el área de conocimiento de Biblioteconomía y Documentación se ha producido en el periodo comprendido entre 1998 y 2008.

4. La difusión de la investigación en innovación educativa en el ámbito de la Información y la Documentación cuenta con canales de difusión todavía poco perfilados.

5. La tarea de rastrear el circuito completo de la innovación educativa en Información y Documentación es compleja debido a la existencia de numerosas zonas de sombra que dificultan la identificación de los agentes, las líneas estratégicas e incluso los resultados, debido a que en 
algunas ocasiones, las evidencias documentales son pocas, dispersas y volátiles.

\section{BI BLI OGRAFÍ A}

Agencia Nacional de Evaluación de la Calidad y Acreditación (2004). Libro blanco. Título de grado en Información y Documentación. Madrid: ANECA, 2004. Disponible en http://www.aneca.es/media/150424/libroblanco jun05 documentacion.pdf Consultado el 11-07-09.

Alonso, Luis Enrique; Fernández Rodríguez, Carlos J. y Nyssen, José María (2009). El debate sobre las competencias: una investigación cualitativa en torno a la educación superior $y$ el mercado de trabajo en España. Madrid: ANECA, Disponible en http://www.aneca.es/media/148145/publi_competencias_090303.pdf . Consultado el 11-07-09.

Aportación a la mejora de la calidad de la docencia universitaria: los mapas conceptuales (2007). Ciencias Sociales, 10. Pamplona: Universidad Pública de Navarra, 2007

Bigs, John (2005). Calidad del aprendizaje universitario. Madrid: Narcea, 2005

Comisión para la renovación de las metodologías educativas en la universidad (2006). Propuestas para la renovación de las metodologías educativas en la Universidad. Madrid: Ministerio de Educación y Ciencia. Secretaría de Estado de Universidades e Investigación, Consejo de coordinación universitaria, 2006. http://profesores.universia.es/docencia/renovacion-metodologias/pdf/propuestarenovacion.pdf . Consultado el 11-07-09

Criterios de clasificación de los medios de difusión de la producción académica y científica universitaria (2007). Madrid: Agencia de Calidad, Acreditación y Prospectiva de las Universidades de Madrid (ACAP), 2007

Gallego Reinoso, Fabio (2005). Aprender a generar ideas. Innovar mediante la creatividad. Paidós aprender, n. 5 Barcelona: Paidós, 2005

Esteve, José M. (2003). La tercera revolución educativa: la educación en la sociedad del conocimiento. Papeles de pedagogía, 61. Barcelona: Paidós, 2003

Martínez, M. A. (coord.) (2004). Investigar en docencia universitaria: redes de colaboración para el aprendizaje. Alcoy: Marfil, (2004)

Papel de la información en la innovación, El (2006). Tecnociencia. Especiales divulgación. FECYT. Disponible en http://www. fecyt.es/especiales/papel informacion/ . Consultado el 10-07-09.

Pulido San Román, Antonio (2008). La Universidad del siglo XXI: Introducción y reflexiones iniciales. En Agencia Nacional de Evaluación de la Calidad y Acreditación. La Universidad del siglo XXI. IX foro ANECA. Madrid: ANECA, 2008, p. 17-24. Disponible en http://www.aneca.es/media/20517/publi 9foro.pdf . Consultado el 11-07-09.

Zabalza Beraza, Miguel Ángel (2002). La enseñanza universitaria. El escenario y sus protagonistas. Madrid: Narcea, 2002.

Zabalza Beraza, Miguel Ángel (2003). Competencias docentes del profesorado universitario. Calidad y desarrollo profesional. Madrid: Narcea, 2003. 